\title{
TI.159.1
}

\section{InCommon Federation SAML 2.0 Profiles; Working Draft 03}

- PDF: InCommon Federation SAML 2.0 Profiles; Working Draft 03

- Text: InCommon Federation SAML 2.0 Profiles; Working Draft 03

\section{More Information}

\begin{tabular}{|l|l|}
\hline Repository ID & TI.159.1 \\
\hline Persistent URL & http://doi.org/10.26869/TI.159.1 \\
\hline Title & InCommon Federation SAML 2.0 Profiles; Working Draft 03 \\
\hline Authors & Scott Cantor, Andreas Åkre Solberg \\
\hline Sponsor & MACE \\
\hline Review & \\
\hline Status & Legacy \\
\hline Publish Date & Feb 18, 2010 \\
\hline DOI & $10.26869 /$ TI.159.1 \\
\hline Signature & \\
\hline Deprecated & \\
\hline Future Review & \\
\hline Supersedes & \\
\hline Format & PDF, text \\
\hline Related Docs & \\
\hline Development Location & \\
\hline IP Framework & CC BY 4.0 \\
\hline Subject Tags & \\
\hline Notes & \\
\hline
\end{tabular}

